\title{
Photon emission and quantum signalling in biological systems
}

\author{
S.N. Mayburov ${ }^{1, a}$ \\ ${ }^{1}$ Lebedev Institute of Physics, Moscow, Leninski pr. 53
}

\begin{abstract}
Ultra-weak, non-termal photon emission is universal feature of living organisms and plants. In our experiment the fine structure of optical radiation emitted by the loach fish eggs is studied. It was shown earlier that such radiation performs the signaling between the distant fish egg samples, which result in significant correlations of their growth. The optical radiation of biological sample was measured by the cooled photomultiplier in photocurrent regime, it was found that the main bulk of radiation is produced in form of short-time quasi-periodic bursts. The analysis of radiation temporal structure indicates that the information about egg age and growth is encoded via the values of time intervals between neighbor bursts with the height higher than some fixed level. The applications of such biological radiation in medical diagnostics and biotechnology are considered.
\end{abstract}

\section{Introduction}

The systematic study of optical and soft UV radiation emitted by living bio-systems continues now for more than thirty years [1,2]; its existence is established now for different biological species [3, 4]. The frequency spectra of such bio-photons is nearly constant throughout optical range for studied bio-systems [5], so it essentially differs from the spectra expected for the system with the temperature about $300^{\circ} \mathrm{K}$, which in this range should fall on 15 orders of magnitude [2,3]. The detailed mechanism of bio-photon production (BP) is still studied, yet it acknowledged that such optical excitations are stipulated mainly by the biochemical reactions, in which oxygen atoms are bound to the proteins and organic acids $[2,3]$.

The typical bio-photon production rates are quite low, however, multiple experiments evidence that such radiation can perform effective signaling between distant bio-systems [2-4]. In particular, being radiated by the growing organism or plant and absorbed by the similar one at the distance about several $\mathrm{cm}$, it can rise the rate of cell division (mitosis) in it up to $30 \%$ relative to the standard values. This phenomenon called mitogenetic effect (ME) is extensively studied in the last years $[2,3]$. Note that the constant illumination of bio-system by the visible light, even $10^{4}$ times more intense can't induce the comparable gain of bio-system development. Communications of some other types were reported also; for bio-systems in the state of abrupt stress or slow destruction (apoptosis) the radiation emitted by it can influence other bio-system in the similar destructive way $[2,6]$.

Until now, ME and some other bio-photon properties can't be explained within standard framework of cellular biology. In our previous paper the model of information exchange between biosystems by means of optical radiation was proposed [7]. We've assumed that the main features of

\footnotetext{
a e-mail: mayburov@sci.lebedev.ru
} 


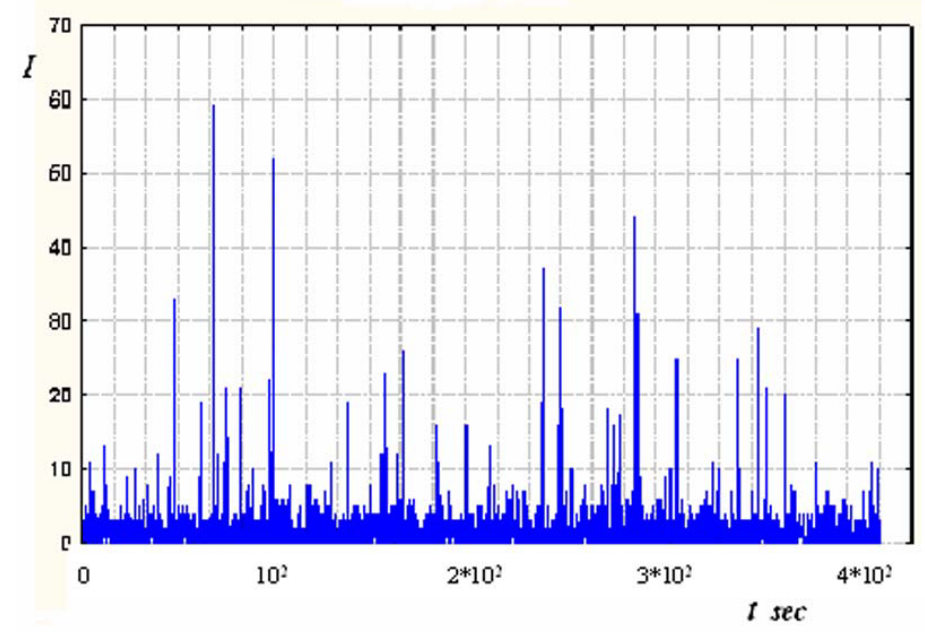

Figure 1. Example of photocurrent $I$ time distribution for fish eggs at development stage 16 , full time scale $4 \cdot 10^{2}$ sec

such signaling can be similar to the information exchange in electronic sircuits. This hypothesis is supported by the recent experiments which show that the radiation of some species consists of narrow quasi-periodic bursts, so its time structure is similar to the sequence of electronic or photonic pulses which transfer information in computer nets $[1,4]$. To verify our model we performed the data analysis for the radiation from loach fish eggs measured by the photomultiplier [1,3]. Here some our results on fine structure of emitted readiation are presented, from their analysis the possible algorithm of photon signal encoding in the fish egg communications is proposed. The production rate and other parameters of bio-photons are quite sensitive to the characteristics of bio-systems which difficult to measure by other methods. Due to it, the bio-photon measurements are applied now in many different fields from medical diagnostics to agriculture and ecology [2]. In particular, below the experiment devoted to the study of bio-photon rates and ME features under conditions of space flight will be described.

\section{Model of Bio-System Communications}

In this section the possible mechanism of bio-photon signaling will be discussed and its semiqualitative model will be considered. First, remind some properties of photon production/absorbtion in the dense media. If the electromagnetic field generated by the system isn't coherent, then with the good accuracy it can be described as the stochastic ensemble of photons [8]. In this case, if the single photon or narrow bunch of photons is absorbed in any dense media, it would induce in it with some probability the short-time excitation of its energy levels. This is the photocounting regime of electromagentic field detection well-known in quantum optics [8]. The standard photodetector device registrates such excitation as the single 'click' corresponding to one bit of information. We suppose that the same approach is applicable also for the photons absorbed in the bio-systems, so that such 'click' can be registrated and memorized by the bio-system also. 


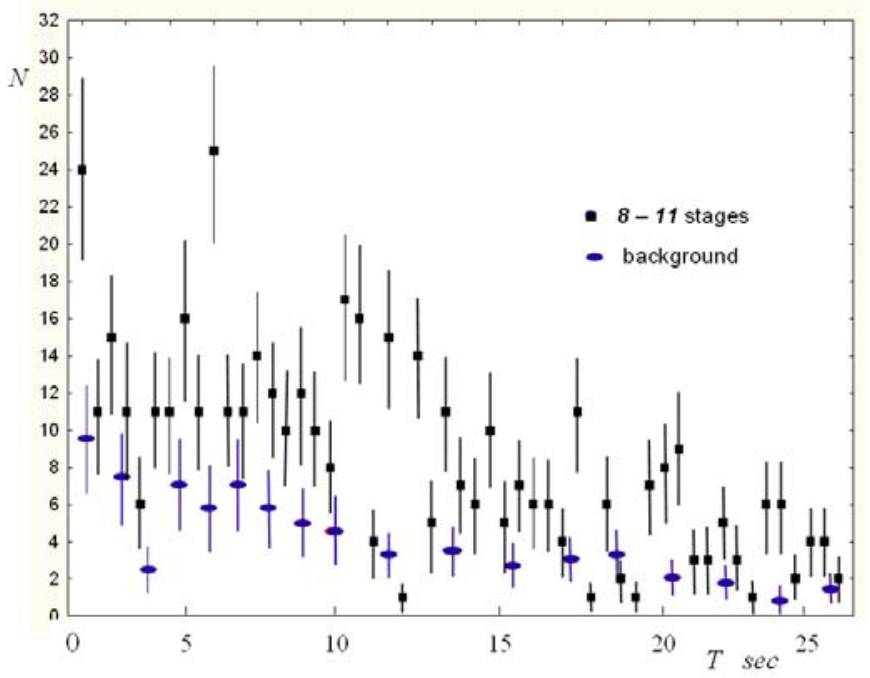

Figure 2. $T$ distributions for fish eggs at $8 \div 11$ stages - full squares and detected background - full circles

As was noticed above, bio-photon rates are quite low, about 10 photons $/ \mathrm{cm}^{2} \mathrm{sec}$ from the surface of large, dense bio-system. Plainly, for such low photon rates the most important problem for effective signaling between distant bio-systems is the background suppression. Even for bio-systems in complete darkness it is produced by many sources, like the natural radioactivity, luminescent chemical impurities, etc.. Consequently, as the criteria characterizing the efficiency of information exchange, the signal to noise ratio $K_{O}$ can be used, where $K_{O}=\frac{\bar{n}_{c}}{\bar{n}_{b}}$, here $\bar{n}_{c}$ is the average ratio of registrated 'clicks' induced by the bio-system signals, $\overline{n_{b}}$ is the radiation background rate. Normally $\overline{n_{b}}$ should be constant in time, so for arbitrary bio-system the optimal method to achieve high $K_{O}$ level is to make the main bulk of the bio-system radiation to be concentrated inside short time intervals, i.e. the bio-system radiation should be produced in form of brief bursts. In this case the burst parameters can encode the signals transferred to other bio-systems. It's natural to suppose that the long-time evolution of living species made the information exchange by means of photon radiation/absorption practically optimal, so the radiation of arbitrary bio-system in general would satisfy the described conditions. The experiments with fish eggs, fibroblast cells and other bio-systems demonstrated that the bio-photons are radiated by short-time (less than $1 \mathrm{msec}$ ) quasi-periodic bursts [1, 4]. As the example, the typical photodetector signal from fish eggs radiation is shown on fig. 1.

The influence of bio-photon exchange on the organism growth and development was found for many species, in particular, their detailed study was performed for the eggs of loach fish ( Misgurnusfossilis). It's well known that for the egg colony produced by the fish during its breeding the maximal rate of larvae survival is achieved, if all eggs develop with the same speed and the free larvae appear during the minimal time interval. However, the small variations of temperature and water flow over colony volume and other external factors tend to violate this condition. The experiments indicate that the signaling between distant eggs of the same colony can restore their simultaneous development. The results for the optical contacts during 60-90 minutes between two samples of fish eggs of slightly different age demonstrate the significant synchronization of their development [1,9]. It was found also that the optical contacts between the fish eggs of significantly different ages result in 


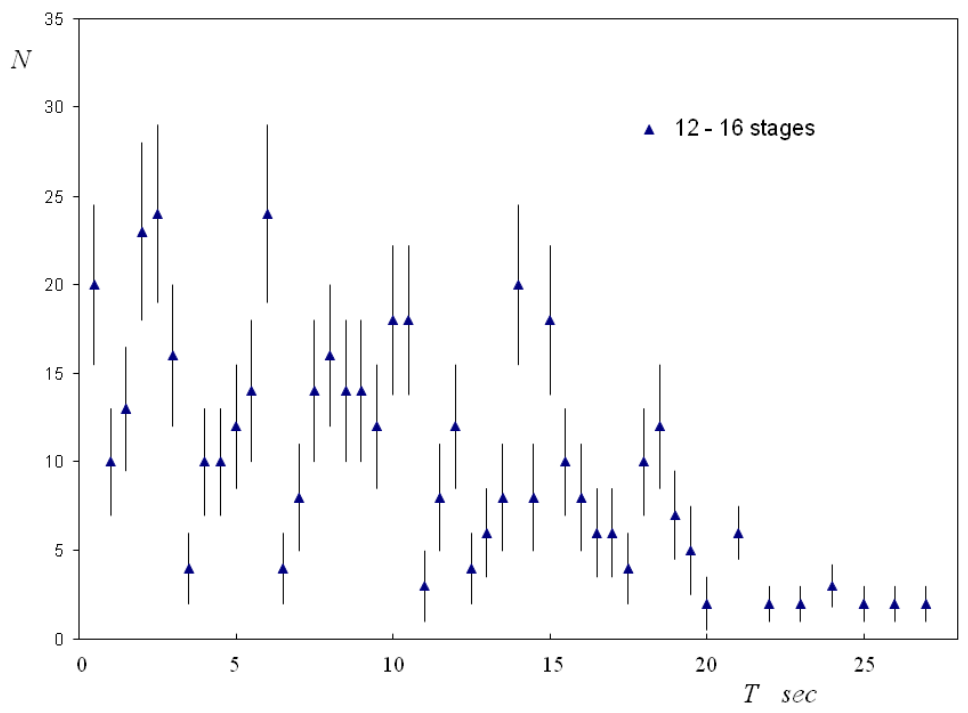

Figure 3. $T$ distribution for fish eggs at $12 \div 16$ stages;

the serious violations of development in both samples, in particular, for fish eggs at early stages their development can simply stop which results in their extinction. Those results evidence that the photon signals emitted by fish eggs of different age can have the essentially different structure, which encode the information about their age and corresponding development program.

Since the radiation background is supposedly stochastic and there is no time correlations between its intensity at different time moments, then the burst periodicity or some other time correlations between them would help to discriminate the background more effectively. Simultaneously, the variations of such correlations can also encode the different signals send by the bio-system. We supposed that such encoding is performed by the algorithms similar to standard ones used for opto-electronic communication channels [7]. In this framework, as the model example, we shall assume that the separate message send by the bio-system on the average consists of $\bar{N}$ bursts of the average height $\bar{I}$ with the average time interval $\bar{T}$ between them, such messages are divided by the periods of bio-system 'silence' which on the average has the duration $\bar{T}_{s}$, presumably $\bar{T}<<\bar{T}_{s}$. During it the detectable radiation corresponds to the background level, so that each separate message is represented the train of bursts divided by $T_{s}$ interval. We shall suppose that at every development stage such identical messages are repeated many times [7]. Plainly, for the realistic bio-systems the burst parameters would have some stochastic spread $\sigma_{I}, \sigma_{T}, \ldots$ around the average values which should be accounted by the model. Below the experimental results for fish egg radiation will be compared with such model template.

\section{Analysis of Experimental Data}

The possible signal structure was exploited for the experimental data on the optical radiation of loach fish eggs [1]. The studied sample is the colony about 200 eggs, which is confined in the quartz container filled with water, the optical and soft UV radiation emitted from the container surface was 


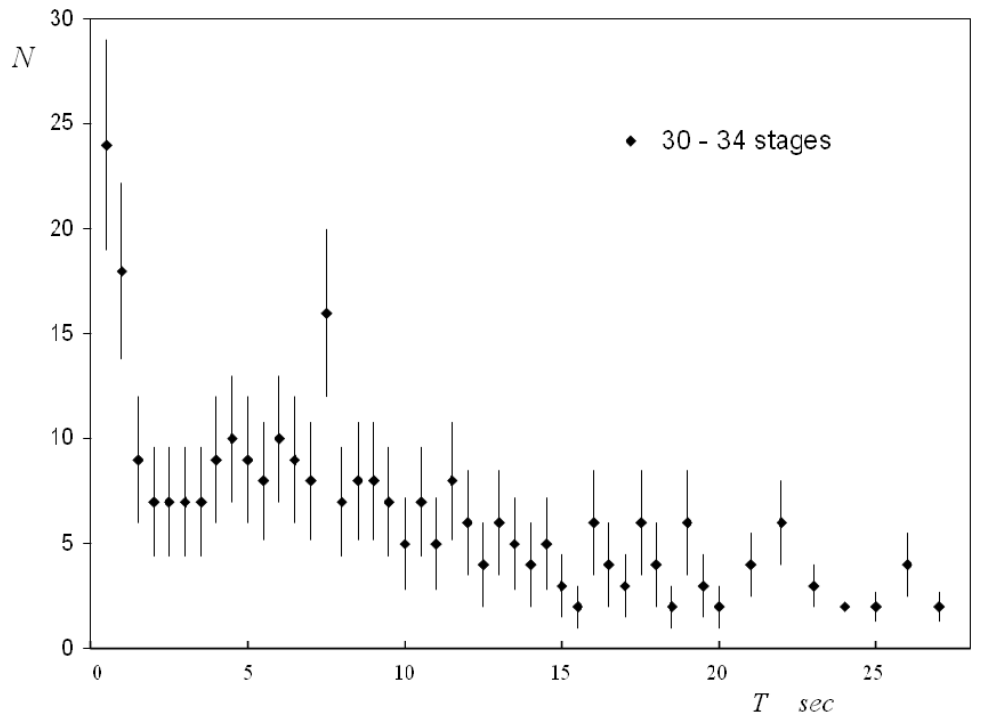

Figure 4. $T$ distribution for fish eggs at $30 \div 34$ stages;

registrated by the photo-multiplier. The resulting photocurrent is summed over the nonoverlapping consequent time intervals (bins) with the duration $\Delta=.1 \mathrm{sec}$; the experimental run normally consists of $6 * 10^{3}$ such bins [1]. The measurements were performed for different development stages, $i$. e. egg age, from the earliest ones (cleavage) to the latest 33-35 stages, preceding the free larvae appearance; the average stage duration is about 1.5 hour. The background radiation was measured for empty container. The collected data were used to extract the possible dependence of detected signal parameters on egg development stage. To reduce the background influence, the registration threshold $I_{0}$ is imposed for the amplitude of selected bursts; it seems plausible that fish eggs can perform the analogous selection.

Concerning the dependence of signal form on development stage, we assumed that the described model parameters can be defined from our data independently of each other. Under this assumption, our analysis indicates that of all these parameters the most pronounced change suffers $\bar{T}$ value. To demonstrate it, $T$ distributions for the bursts with $I$ higher than $I_{0}=15$ units (see fig. 1) were obtained for several development stages. To enlarge statistics, we summed in the same plot the data for $8-11$, $12-16$ and 30-34 stages. Under these conditions the total exposition time for each plot was $130 \mathrm{~min}$. Fig 2. shows that for the same exposition time the detected background is essentially less intensive than the fish egg radiation at $8-11$ stages. The same is true also for radiation intensity at $12-16$ and $30-34$ stages shown on fig. 3 and 4 correspondingly.

The obtained $\bar{T}$ expectation values are equal to $\bar{T}=11.2 \pm .3,9.2 \pm .3$ and $7.1 \pm .2$, sec for $8 \div 11$, $12 \div 16$ and $30 \div 34$ stages correspondingly. Meanwhile, the average number of bursts is nearly the same for all three cases, whereas the average burst amplitude $\bar{I}$ for $30-34$ stages is about $15-20 \%$ larger than for $8-11$ stages, yet the overlap of their $I$ distributions is essentially larger than for $T$ distributions. For other model parameters $\bar{N}, \bar{T}_{s}$ the collected statistics doesn't permit to obtain the reliable estimates of their values, however on the qualitative level the high bursts seems to constitute the clusters interspersed by the periods of 'silence'. The essential feature of all three plots is the 


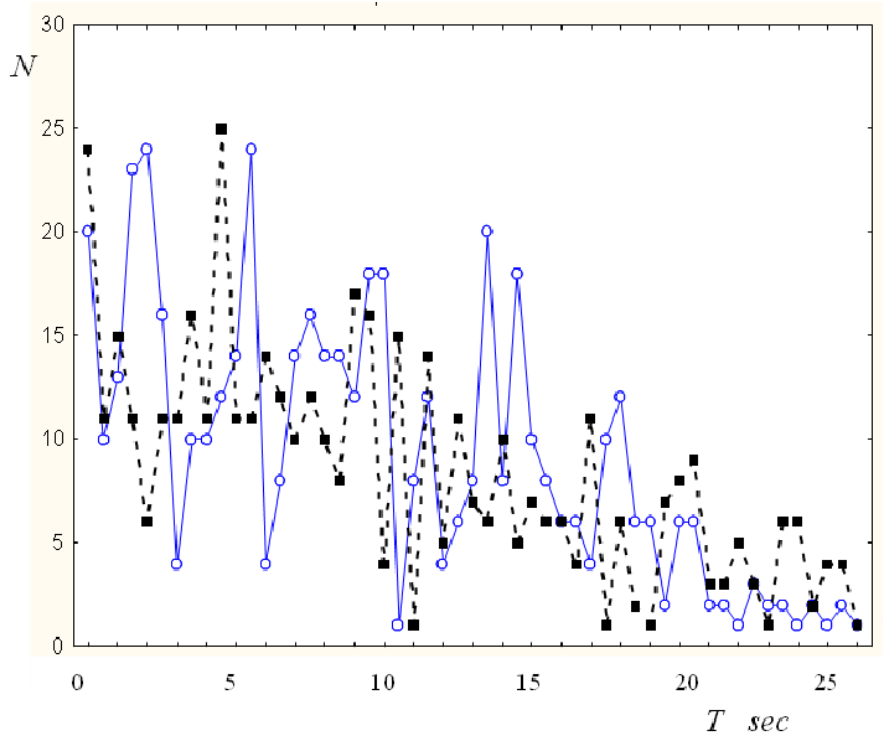

Figure 5. Fits of $T$ distributions for fish eggs radiation; broken line: $8 \div 11$ stages; solid line $12 \div 16$ stages

presence of several large peaks, which are effectively higher than the possible statistical error limits. Their parameters need further analysis, but it's worth to notice that for $8-11$ and $12-16$ stages two most prominent peaks seems to be generically connected, shifting from 5.0 to $6.1 \mathrm{sec}$ and from 10.3 to $10.8 \mathrm{sec}$ ( see fig. 5). Note also that $T$ maxima positions of three largest peaks for $8-11$ stages are related approximately as $1: 2: 4$.

The obtained distinctions explain, probably, how the radiation from fish eggs of different age can influence the 'detector' samples in a different way. Despite that the difference of signal parameters is only statistical, the multiple repetition of such signals can be eventually percepted by the 'detector' fish eggs as the different instructions which would be fulfilled during their subsequent development. It seems that the most important for their encoding is $T$ difference, because the burst amplitude produce mainly the threshold effect, i.e. only the condition like $I>I_{0}$ is accounted for some arbitrary $I_{0}$ which is proper for given bio-system. If those conclusions will be confirmed by further experiments, it would mean that the main encoding algorithm for fish eggs radiation has the analogue realization, despite that the produced signal is constituted by the sequence of discrete bursts. Such information encoding is similar to the digital-time analogue (DTA) algorithm used in standard electronic sircuits. Note that the similar encoding of electric pulses supposedly exists in the brain neuron chains [10].

\section{Bio-photon Measurements in Space; Orlando Project}

As was noticed above, the bio-photon measuremnts now has many applications, especially, in medical diagnostics, ecology and bio-technology. In particular, they can be exploited for noninvasive monitoring of biotechnological processes and bacteria growth. This topics are especially important for long-time space missions. To study their realization by ORLANDO project it's planned to compare bio-photon effects in lab. conditions and on the earth orbit. For that the specialized module will be delivered to International Space Station (ISS). In this set-up the array of 7 photomultipliers will 
measure photon radiation of different biological samples during their lifecycle, for that purpose the photocurrents during several days will be recorded with high time resolution. ME for bio-systems will be also studied performing optical contact of inductor and detector samples at the distance about $10 \mathrm{~cm}$. During first series of experiments photon radiation of bacteria cultures (sacharomicetes) will be studied; experiments with other biological species are considered for the next series of flights. One of experimental program aims is to investigate the mechanism of anomalous bacteria growth found during previous orbital flights. Study of bio-photon rate dependence on astrophysical factors, like Sun and Moon cycles, observed earlier in lab. conditions [14], is also planned.

\section{Discussion}

Described data analysis presumably confirm our model of photonic communications between distant bio-systems. The obtained results can help to establish the mechanism of communications between the distant samples of fish eggs and permit to describe the universal features of bio-photon signaling between the separate bio-systems and distant parts of the same organism. Remind that the optical excitations in the dense media can exist as the quasi-particles called excitons which can spread freely through the whole media volume [11]. They are strongly coupled with electromagnetic field, so they can be effectively produced during the photon absorption by the media, the inverse process results in the photons emission from the system surface. It's established experimentally now that the excitons play the important role in the energy transfer inside the bio-systems, in particular, during the photosynthesis in plants and bacteria $[10,12]$. We don't consider here any particular dynamics of such processes, however, for such large distances it's inevitably should have the solitonic properties [11]. In this vein it can supposed that the excitons spread freely over all the volume of bio-system. In that case the exciton exchange can constitute the effective system of signaling and regulation of the bio-system development. We suppose also that the excitations of biological media in form of excitons play the crucial role in bio-photon generation and absorption by the bio-system which result in ME. There are also the multiple evidences that the long-distance signaling takes place between the distant parts of the same organism [7]. In particular, the experiments evidence that such long-distance signaling regulates effectively the plant growth, preventing from the large fluctuations of its global form, i.e. defines their morphogenesis [2, 3].

The cell signaling and regulation features are well studied for the extracellular biochemical reactions [10]. Concerning the chemical signaling in the tissues, its efficiency and precision is principally restricted by the molecular diffusion effects inside the bio-system media and so can transfer the signals only for small distances. Note also that the exciton signaling inside organism can be much faster, than the chemical one by means of molecular messengers. Hence it can be efficient in case of stress or the abrupt change of external conditions. Experimental results show that under the different stress conditions the photon rates from bio-system can rise in short time significantly, probably, as the consequence of intensive internal signaling [2,3].

Our model assumes that the bio-system's field is noncoherent, yet it's worth to consider also the possibility that this electromagnetic field of bio-system can possess the spacious short-time coherence within the observed photon bursts, similarly to the coherence of laser pulses. Some experiments evidence that such bio-photon coherence really takes place [13]; in this experimental set-up the transparent quartz plate was installed between the inductor and detector bio-systems. In the first run the plate parallel surfaces were smooth and polished, so that it doesn't perturb the phase relations between the different pieces of wave front of incoming photons. In another run, the plate has the random deflections from the surface parallelism, which violated such phase relations, and so can destroy the impact wave coherence. It was found that in comparison with the control sample of isolated bio-system, the 
radiation passed through the random surface results in the gain of mitosis rate of $20 \%$, yet for smooth surface it reach the rate of $45 \%$. In our model framework, it's reasonable to suppose that the field with such 'transversal' coherence more effectively produce the collective excitations of bio-system, than noncoherent field. If this hypothesis is correct, then such coherence effects would not change the principal scheme of communications proposed here, rather, it would enlarge its efficiency.

Author is thankful L.V. Beloussoff and I.V. Volodiaev for providing the experimental data files and the extensive consultations on related topics.

\section{References}

[1] Beloussov, L.V., BioSystems 68, 199-212 (2003)

[2] VanWijk, R., J. Sci. Explor. 15, 183-209 (2001)

[3] Popp, F.A. et al., Collect. Phenomena 3, 187-198 (1981)

[4] Beloussov, L.V. , Burlakov, A.B., Ind. J. Exp. Biol., 41,424-430 (2003)

[5] Tilbury R.N., Quickenden T.I., J. Bioluminescence 7, 245-253 (1992)

[6] Farhadi, A. et al., Bioelectrochemistry 71,142-148 (2008)

[7] Mayburov, S., Quant. Comp. Comp. 11, 73-81 (2011)

[8] Glauber, R.J., Quantum Optics (Academic Press, N-Y, 1969) 56-74

[9] Burlakov A.B. et al., Ontogenesis 31,340-348 (2000)

[10] Shubin A.F., Biophysics (Moscow, Nauka, 1998), 165-189

[11] Davidov A., Solitons in Molecular Physics (Kluwer, Dortreht, 1991) 63-96

[12] Engel G.S. et al., Nature 446, 782-787 (2008)

[13] Budagovsky A., In: Biophotonics and Coherent Systems in Biology. (Springer, Berlin, 2007) 81 $-94$

[14] Gallep C.M. et al., Protoplasma 250 793-796 (2012) 